\title{
REAL TIME DROWSINESS DETECTION USING DIFFERENT EDGE DETECTION TECHNIQUES
}

\author{
Gururaja A K ${ }^{1}$, K.V.Mahendra Prashanth ${ }^{2}$ \\ ${ }^{I}$ Student, Digital Electronics, M.Tech, SJBIT, Karnataka, India \\ ${ }^{2}$ Professor, ECE Department, Chief Coordinator, R\&D and PG Programmes, SJBIT, Karnataka, India
}

\begin{abstract}
Irrespective of the advances in automobiles and roadway development a substantial extent of road accidents exist still because of driver drowsiness. Edge detection is a vital preprocessing image processing and computer vision technique. In the paper, a nonintrusive vision based frame work is employed for identification of car driver tiredness. Real time images have been considered for the experiments. Various edge detection techniques such as Sobel edge, Canny edge, Prewitt's and Robert's edge detection technique have been employed to detect the edges of the eye and determine the number of times the eye closes and opens. The results reveals that Sobel edge detection algorithm is easy to design as compared to other edge detection techniques. The output of Sobel algorithm demonstrates the proposed algorithm is more effective for edge recognition than other edge detection algorithms. The objective of the work carried out is design and development of a drowsiness detection system with more accuracy to reduce the incidents of road accidents.
\end{abstract}

Keywords: Drowsiness Detection, Edge Detection; Sobel Operator, Canny, Prewitt and Robert Operators. $* * *$

\section{INTRODUCTION}

Large quantities of vehicle accidents are occurring due to car driver drowsiness. The main reasons for accidents are driver's drowsiness, while driving. Number of vehicles are increasing day by day, there are around 90+Lakh vehicles (other than two wheeler) in the city. In this paper the system measures, mainly safety and to provide safety, the driver should be alert on the road at day time and during night. The main motivation is to design this proposed system to decrease driving accidents using the computer vision tool and image processing.

In the history of the computer vision and Image processing, the edge detection is a method which tries to capture the important things of objects in the image. The proposed system utilizes a multicolored video camera which points to the car user's face and displays the car user's eye in order to sense micro-sleeps. The proposed system is used to search for the face in the input using the skin color details [3]. Then it performs the process to get exact position of the face, by separating the pixels with skin like color. Similar technique is used to detect whether the eyes are open or close, by tracking the location of the pupil.

\section{RELATED WORKS}

A versatile learning technique for continuous car driver eyes detection, which may perhaps be utilized for detecting a car driver's alertness limit. This paper presents an adaptive learning method for real-time driver eye detection, which could be used for monitoring a driver's vigilance level while he/she is operating a vehicle on road.[1].
Fatigue is one of the principle reasons of negligence of driver's in the driving. Paper [2] deal with ways to identify fatigue of car user. The system distinguishes the face from a video sequence arrangement of car driver's anterior face, follow on finding the eye from the detected face. Besides, the framework finds the presence of eye understudy from recognized eye and measures the blink rate all the while and by dividing these parameters, the framework measures the loss of mindfulness before driver totally loses his or her concentration. In the paper[4], the fatigue can be recognized by utilizing the sensors, which are set close to driver eyes for flicker recognition. This includes distinguishing the eye flicker conclusion and looking return through infra-red sensor. Then the framework will give a bell signal and the pace of the vehicle is decreased.

This paper proposes a well-defined system to defeat the above issues, by representing a computer vision framework for constant observation of driver's drowsiness. It depends on a framework, for continuous monitoring of driver's images utilizing a dynamic camera illuminator, and their product usage for observing some visual practices that represents a driver's level of drowsiness.

\section{PROPOSED METHODOLOGY}

Sleepy driving is a significant transportation security concern and it leads to a huge number of accidents. The subsequent damages of inactive driving could be much higher among in the business vehicles. If the driver is tired, driver' loses his control, prompting unpredicted vehicle direction. Dependable security frameworks are expected to dismiss these accidents. The most vital test is to distinguish the driver's condition adequately ahead of schedule, preceding the onset of rest, to stay away from crashes. 
Different identification strategies have been proposed by specialists and a couple of frameworks are accessible in the business market. The main objective is to design and develop Sobel edge detection, Robert, Prewitts and Canny edge detection algorithms to detect the eye region in the driver's face. The comparative evaluation of percentage of drowsiness reveals that Sobels edge detection algorithm provides better performance as compared to other methods. The algorithm has been developed using MATLAB using computer vision toolbox.

\subsection{SobelAlgorithm}

The Sobel edge detection technique employs two $3 \times 3$ matrix kernels, those are convolved with the original image to produce-one for horizontal changes and one for vertical. On the other hand, the system considers Bas the input image, $\mathbf{H x}$ and Hy are 2 matrix pictures, where every point contains the horizontal and vertical subordinate approximations, the procedure to calculate the derivatives are given as per the following.

$$
\mathbf{H x}=\left[\begin{array}{lll}
-1 & 0 & +1 \\
-2 & 0 & +2 \\
-1 & 0 & +1
\end{array}\right] * \boldsymbol{B} \text { and } \mathbf{H y}=\left[\begin{array}{ccc}
-1 & -2 & -1 \\
0 & 0 & 0 \\
+1 & +2 & +1
\end{array}\right] * \boldsymbol{B}
$$

Where, two-dimensional convolution process indicated by *.

Since the Sobel kernels can be decomposed as the products of an averaging and a differentiation kernel, they compute the gradient with smoothing.

From the outcome matrix of convolution the y-direction is characterized as expanding in the "down"- bearing and $\mathrm{x}$ direction is characterized here as expanding in the "right"course. From every point in the image, the gradient magnitude can be obtained by merging the resulting gradient approximation.

$$
\mathbf{H}=\sqrt{\mathbf{H} \mathbf{x}^{2}+\mathbf{H} \mathbf{y}^{2}}
$$

Utilizing this data, we can likewise compute the gradient's direction:

$$
\sum(\mathbf{x}, \mathbf{y})=\arctan (\mathbf{H y}(\mathbf{x}, \mathbf{y}) / \mathbf{H x}(\mathbf{x}, \mathbf{y}))
$$

\subsection{Roberts Algorithm}

Roberts edge detection algorithm is one of the main and first edge finders. The Roberts cross algorithm is used to estimate the gradient of an image done through discrete differentiation which is attained by doing the sum of the squares of the differences between opposite diagonally adjoining pixels.

Convolute the input image, with the following two kernels:

$$
\left[\begin{array}{cc}
-1 & 0 \\
0 & -1
\end{array}\right] \text { and }\left[\begin{array}{cc}
0 & +1 \\
-1 & 0
\end{array}\right]
$$

Let the outcome after convolving with the first kernel is $\mathbf{H}_{\mathbf{x}}(\mathbf{x}, \mathbf{y})$ be a point in an image formed. The outcome by convolving with the second kernel is $\mathbf{H}_{\mathbf{y}}(\mathbf{x}, \mathbf{y})$ be a point in an image formed. $\mathbf{I}(\mathbf{x}, \mathbf{y})$ be a point in the input image. The gradient can then be defined as:

$\nabla \mathbf{I}(\mathbf{x}, \mathbf{y})=\mathbf{H}(\mathbf{x}, \mathbf{y})=\sqrt{\mathbf{H x}^{2}+\mathbf{H y}^{2}}$

The direction of the gradient:

$$
\sum(\mathbf{x}, \mathbf{y})=\arctan (\mathbf{H y}(\mathbf{x}, \mathbf{y}) / \mathbf{H x}(\mathbf{x}, \mathbf{y}))
$$

\subsection{Prewitts Algorithm}

The Prewitt algorithm is mainly used as a part of image processing, in specifically edge detection calculations. Actually, it is a discrete differentiation administrator, doing an estimation of the gradient of the image intensity function. Prewitt administrator result produces either to relating gradient vector or the standard of this vector done by at every point in the image. Then in the similar way of convolving like other algorithms, the Prewitt algorithm performs convolving the image with filter in horizontal and vertical directions and hence it is generally economical as far as calculations are concerned.

Mathematically, the algorithm employs two $3 \times 3$ matrix which are mainly used for convolving with the input image to determine approximations of the derivatives, one for horizontal and one for vertical. On the other hand that system considers Bas the input image, $\mathbf{H x}$ and $\mathbf{H y}$ are two images which at every point contain the horizontal and vertical derivatives

$$
\mathbf{H} \mathbf{x}=\left[\begin{array}{lll}
-1 & 0 & +1 \\
-1 & 0 & +1 \\
-1 & 0 & +1
\end{array}\right] * \boldsymbol{B} \text { and } \mathbf{H y}=\left[\begin{array}{ccc}
-1 & -1 & -1 \\
0 & 0 & 0 \\
+1 & +1 & +1
\end{array}\right] * \boldsymbol{B}
$$

Where, two-dimensional convolution process indicated by *.

From the outcome matrix of convolution the y-direction is characterized as expanding in the "down"- bearing and $\mathrm{x}$ direction is characterized here as expanding in the "right"course. Every point in the image, the gradient magnitude can be obtained by merging the resulting gradient approximation.

$$
\mathbf{H}=\sqrt{\mathbf{H} \mathbf{x}^{2}+\mathbf{H y}^{2}}
$$

Using this information, we can likewise calculate the gradient's direction:

$\sum(\mathbf{x}, \mathbf{y})=\arctan (\mathbf{H y}(\mathbf{x}, \mathbf{y}) / \mathbf{H x}(\mathbf{x}, \mathbf{y}))$

\subsection{Canny Algorithm}

The canny edge algorithm is an edge detection technique that utilizes a multi-stage calculation to distinguish an extensive variety of edges in images. Canny edge recognition is a strategy to separate valuable basic data from 
various vision objects in different computer vision frameworks. This technique mainly works in the application of edge detection where it has relatively similar small edges. In this manner, an edge identification technique can be actualized in an extensive variety of situations for different locations.

The Procedure of canny edge location can be separated to 5 diverse strides:

1. Apply Gaussian channel to smooth the image.

2. Find the image's intensity gradient.

3.Non-maximum suppression method is applied to get rid of false response of the edges.

4. Apply threshold to decide possible edges

5.Track edge of the image by using the hysteresis: Conclude the position of edges by suppressing the various edges that are not strong and are not associated with strong edges.

As edge recognition is a basic step in computer vision, it is important to appeal consideration to the genuine edges to get the best results from the coordinating procedure. In this paper, the system first presents some main points and impediments of edge identification systems.

Table -1: Advantages and disadvantages of edge detection algorithms

\begin{tabular}{|l|l|l|}
\hline Algorithms & Advantages & Disadvantages \\
\hline $\begin{array}{l}\text { Sobel, Prewitts, } \\
\text { Robert }\end{array}$ & $\begin{array}{l}\text { Simple to design, } \\
\text { orientations of } \\
\text { edges and their } \\
\text { Identification. }\end{array}$ & Noise sensitive \\
\hline Canny & $\begin{array}{l}\text { Increasing the } \\
\text { signal to noise } \\
\text { ratio, especially } \\
\text { in noise } \\
\text { situations, } \\
\text { Localization, } \\
\text { Better detection }\end{array}$ & $\begin{array}{l}\text { Complex } \\
\text { Computations, } \\
\text { Time consuming }\end{array}$ \\
\hline
\end{tabular}

\section{EXPERIMENTAL REULTS}

\section{PERFORMANCE ANALYSIS}

The proposed drowsiness recognition framework utilizing Computer vision method is presented.

The yield chart of edge detection algorithm is demonstrated as follows. Every cycle ( 7 iterations) is prepared and demonstrates the outcomes, if the individual close his eyes in the 5 outlines ( 5 iterations) out of 7 iterations, then proposed system conclude that driver is in drowsiness.

The percentage of drowsy can be calculated by using the below formula.

Percentage of Drowsy $=\frac{\text { No of times eye closed }}{\text { Total no of frames }} * 100$
Table -2: Experimental outcomes

\begin{tabular}{|l|l|l|l|l|}
\hline Persons & $\begin{array}{l}\text { Video } \\
\text { length }\end{array}$ & $\begin{array}{l}\text { Eye } \\
\text { open }\end{array}$ & $\begin{array}{l}\text { Eye } \\
\text { close }\end{array}$ & $\begin{array}{l}\text { Percentage } \\
\text { of Drowsy }\end{array}$ \\
\hline 1 & 10 frames & 4 & 6 & 60 \\
\hline 2 & 20 frames & 12 & 8 & 40 \\
\hline
\end{tabular}

If the percentage of drowsiness is more than $60 \%$, then the driver is in drowsiness state.

\section{A. Sobel Algorithm}

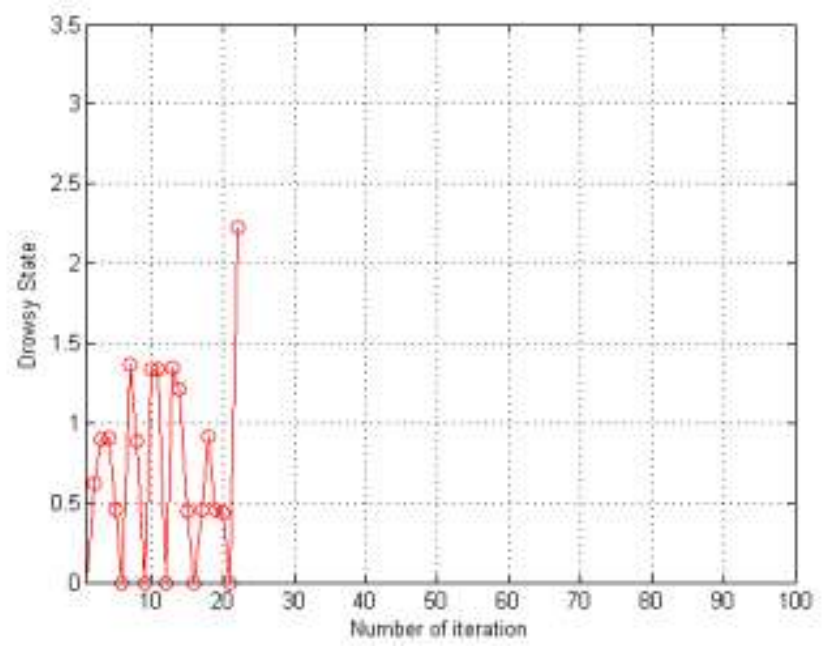

Fig -1: Sobel Algorithm $($ Threshold = 0.2)

Figure1 shows the output of Sobel operator (Sobel Algorithm (Threshold $=0.2)$ ), which is processed in Mat lab and each cycle represented as a bubble. Here the graph is in wave shape, it is obtained by joining the bubbles, where each bubble is the outcome of the 7 iterations. The 7 iterations comprise a cycle. If the drowsy state is below 1 $(<1)$ then the driver is not in drowsiness. If the graph is in between 1 and $2(1<$ output<2), then the driver is in partial drowsiness. In the similar way, if the drowsy state above 3 $(>3)$, then the driver is in drowsy state.

\section{B. Roberts Algorithm}

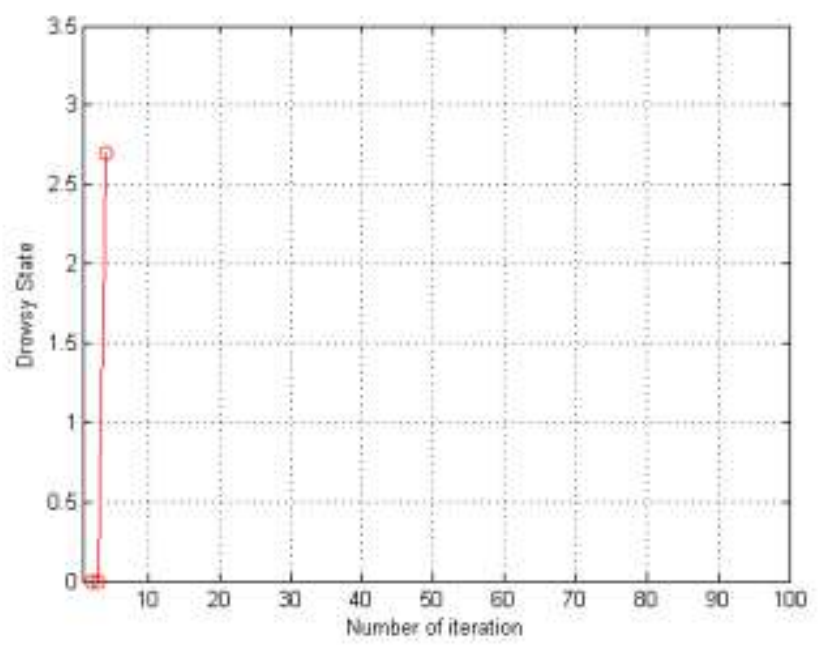

Fig -2: Roberts Algorithm (Threshold = 0.2) 
Roberts edge identification algorithm is same as the Sobel edge detection algorithm, and uses $2 \times 2$ kernel instead of $3 \times 3$, in contrast to Sobel algorithm, Hence the yield of the Roberts additionally varies from the Sobel algorithm.

\section{Prewitts Algorithm}

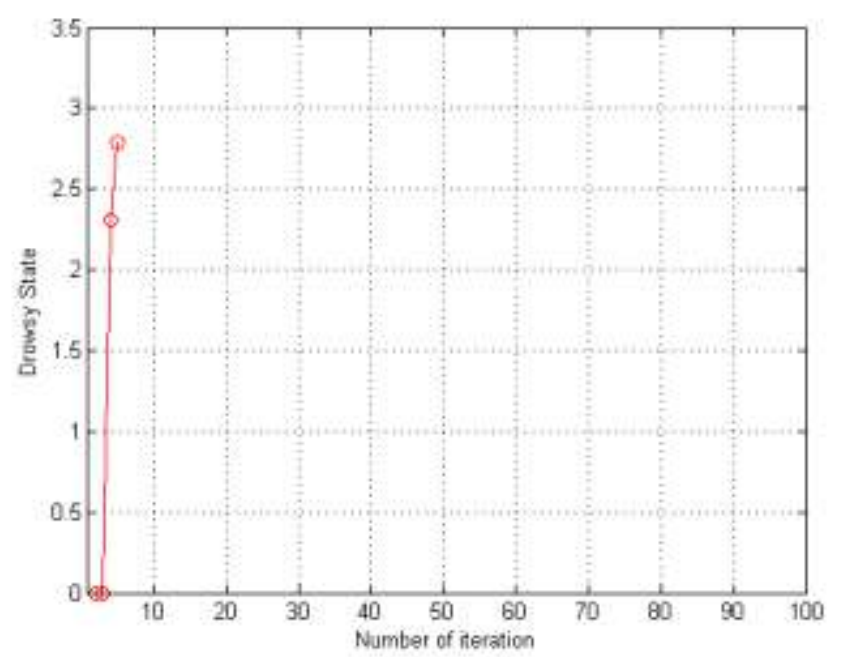

Fig -3: Prewitts Algorithm (Threshold = 0.2)

Fig 3 shows the drowsiness detected using Prewitt's edge detection algorithm.

\section{Canny Algorithm}

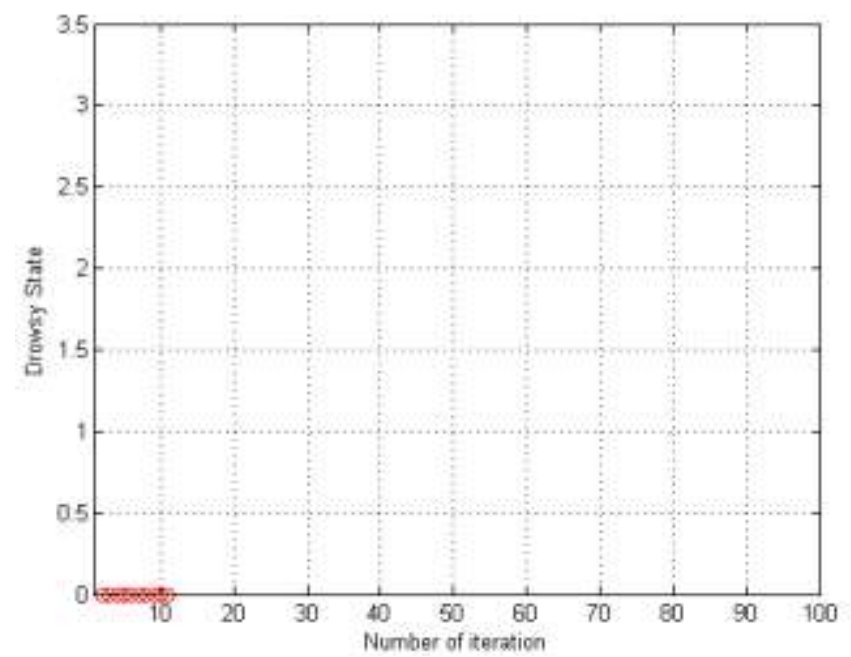

Fig -4: Canny Algorithm (Threshold = 0.2)

The results of drowsiness detection obtained through Canny edge detection is shown in fig 4 . The results shows that Sobel edge detection achieves better detection of drowsiness state in less computation time as compared to Prewitt's and Roberts edge detection techniques.

\section{CONCLUSION}

The designed system has addressed different edge detection techniques such as Sobel, Canny, Prewitt's and Robert's to detect the drowsiness of the driver. In this paper drowsiness detection framework based on the computer vision tool is employed. The system computes the edge detection technique to find out the edges in the eye region by using different edge detection algorithms. The results reveals that Sobel provides better accuracy of drowsiness detection with faster computation as compared to other edge detection techniques such as Robert's, Canny and Prewitt's edge detection techniques.

\section{REFERENCES}

[1] Zhang Guang-yuan, Cheng Bo Feng Rui-Jia, Zhang XiBO "A Real-Time Adaptive Learning Method for Driver Eye Detection," IEEE Transactions on Electronics Packaging Manufacturing, 2008, pp. 300304.

[2] ManashChakraborty and AhamedNasif Hossain Aoyon "Implementation of Computer Vision to Detect Driver Fatigue or Drowsiness to Reduce the Chances of Vehicle Accident," International Conference on Electrical Engineering and Information \& Communication Technology (ICEEICT) and IEEE, 2014.

[3] Sarbjit Singh Nikolaos and P. Papanikolopoulos "Monitoring Driver Fatigue Using Facial Analysis Techniques," IEEE, 1999, pp. 314-318.

[4] B.Praveenkumar and K.Mahendrakan "Prevention of Accident Due To Drowsy By Using Eye Blink," International Journal of Innovative Research in Science, Engineering and Technology, may 2014, vol. 3, pp. 12610-12617.

[5] W.B. Hong, C.Y. Chen, Y. Chang, and C.H. Fan, "Driver fatigue detection based on eye tracking and dynamic template matching," in Proceedings of the 2004 IEEE International Conference on Networking, Sensing \& Control, 2004, pp. 7-12.

\section{BIOGRAPHIES}

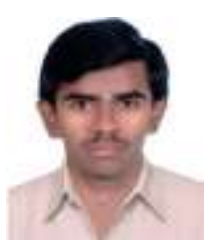

Gururaja A K received the B.E. degree in Electronics and communication from the Global Academy of Technology under Visveswaraya Technological University, Belagavi, Karnataka, in 2014, and the M.Tech in Digital Electronics (Persuing) from SJBIT under Visveswraya Technological University, Belagavi, Karnataka.

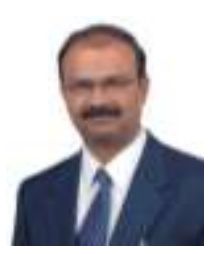

Dr. K.V. Mahendra Prashanth has obtained his B.E. degree in Electronics \& Communication Engineering at National Institute of Engineering, Mysore; M.E. degree in Power Electronics from UVCE Bangalore. He received his Ph.D. degree in Electronics \& Communication Engineering from Visvesvaraya Technological University, Belagavi. His area of interests are signal processing, active noise control, Bio-Medical engineering. He has around 23 years of teaching experience. Presently, he is working as Professor in Department of Electronics \& Communication Engineering, Chief Coordinator for R\&D and PG programmes at SJBIT, Bangalore. He has published several papers in international and National journals, and presented papers in International conferences. He is a Reviewer for international \& National Journals. He is the Member of IEEE, Acoustical society of India, ACCS, IIAV, ISTE etc. 